\title{
On-chip electrochemical detection of glucose towards the miniaturised quality control of carbohydrate-based radiotracers
}

\author{
Laila Patinglag, ${ }^{a, s, 0}$ Mohammad M. N. Esfahani, ${ }^{a, b, b}$ Kishan Ragunathan, ${ }^{a}$ Ping He, ${ }^{a, c}$ \\ Nathaniel J. Brown, ${ }^{\mathrm{b}}$ Stephen J. Archibald, ${ }^{\mathrm{a}, \mathrm{c}}$ Nicole Pamme ${ }^{\mathrm{a}}$ and Mark D. Tarn ${ }^{\mathrm{a}, \mathrm{c}}$
}

\begin{abstract}
The miniaturisation of positron emission tomography (PET) radiotracer production is facilitating a move towards a doseon-demand strategy that would enable a stratified approach to patient diagnostics, but while the on-chip synthesis steps have been demonstrated, the subsequent quality control (QC) testing steps have received much less attention. As part of the development of an integrated QC platform for PET tracers, we have developed two microfluidic electrochemical detectors for the pulsed amperometric detection (PAD) of carbohydrate-based radiotracers, with a particular view to the $\mathrm{QC}$ testing of the most important tracer, $\left[{ }^{18} \mathrm{~F}\right] 2$-fluoro-2-deoxy-D-glucose $\left.\left({ }^{18} \mathrm{~F}\right] \mathrm{FDG}\right)$. The first device employed a commercial screen-printed electrode (SPE) to enable a single-use format, while the second device incorporated wire electrodes for use as a more permanent fixture in a QC instrument. A flow-injection analysis (FIA)-style setup was used to inject boluses of D-glucose into the chips in a proxy for intended chromatographic separations prior to PAD. In proof-ofconcept testing of the devices, the chips featuring the SPE and the wire electrodes yielded limits of detection of $0.1 \mathrm{ppm}$ and $9 \mathrm{ppm}$, respectively, each below the required limits for $\left[{ }^{18} \mathrm{~F}\right] \mathrm{FDG}$, and thus making both methodologies viable for the QC testing of PET radiotracers in a dose-on-demand format.
\end{abstract}

\section{Introduction}

Positron emission tomography (PET) medical imaging ${ }^{1}$ may soon be revolutionised via the introduction of dose-ondemand radiopharmaceutical production. ${ }^{2-9}$ In the current "centralised" methodology, large batches of a single type of radiotracer, usually $\left[{ }^{18} \mathrm{~F}\right] 2$-fluoro-2-deoxy-D-glucose $\left(\left[{ }^{18} \mathrm{~F}\right] \mathrm{FDG}\right)$, are synthesised at a production facility before being transported to an imaging centre for administration to patients prior to their PET scan. However, dose-on-demand or "decentralised" production would allow single doses of specific tracers to be generated at the imaging centre, requiring only transport of the radioisotope (if local generation is

\footnotetext{
${ }^{a .}$ Department of Chemistry and Biochemistry, University of Hull, Cottingham Road, Hull, HU6 7RX, UK.

Department of Engineering, University of Hull, Cottingham Road, Hull, HU6 7RX, UK.

b. Positron Emission Tomography Research Centre, University of Hull, Cottingham Road, Hull, HU6 7RX, UK.

$\S$ Current address: Faculty of Science and Engineering, Manchester Metropolitan University, Chester Street, Manchester, M1 5GD, UK.

‡ Current address: School of Earth and Environment, University of Leeds, Woodhouse Lane, Leeds, LS2 9JT, UK.

*E-mail: m.d.tarn@leeds.ac.uk; Tel: +44 (0) 1133435605.

$\checkmark$ Authors contributed equally to this work.

+ Footnotes relating to the title and/or authors should appear here.

Electronic Supplementary Information (ESI) available: Details of the screen-printed electrodes (SPEs) and wire electrodes. Setup of the electrodes, microfluidic devices and flow injection analysis (FIA) system. Data from tests of the SPE-chip usin potassium ferricyanide. Data showing the effects of varying the pulsed amperometric detection (PAD) waveform potentials. See
} DOI: $10.1039 / x 0 x x 00000$ unavailable), e.g. $\left[{ }^{18}\right.$ F]fluoride, for on-site synthesis. This strategy will enable greater flexibility in radiotracer production, allowing tracers to be synthesised on a patient-bypatient or stratified basis. Microfluidic technology ${ }^{10}$ is crucial to the success of dose-on-demand, but while microreactors ${ }^{9,20-}$ ${ }^{33}$ have been employed for small-scale synthesis of a number of PET radiotracers, many of the important follow-up steps have been largely ignored. For example, there are only a handful of examples of microfluidic devices developed to address the myriad of stringent quality control (QC) tests required prior to the release of tracers for use in human patients. ${ }^{11-13}$

A number of $Q C$ tests are required to confirm that levels of potential contaminants are below or within certain thresholds, and these include the analysis of $\mathrm{pH}$, sterility, residual solvents, catalysts, radioisotope identity and purity, and radiochemical identity and purity. ${ }^{11,14,15}$ The latter two tests are conventionally achieved using chromatographic techniques, ${ }^{16}$ as specific by the British Pharmacopoeia (BP), ${ }^{17}$ European Pharmacopoeia (EP), ${ }^{18}$ United States Pharmacopeia (USP), ${ }^{19}$ the International Pharmacopoeia published by the World Health Organization (WHO), ${ }^{20}$ and the Chemistry, Manufacturing, and Controls (CMC) Guidances published by the Food and Drug Administration (FDA). ${ }^{21}$

One of the tests for confirming the radiochemical identity and purity of $\left[{ }^{18} \mathrm{~F}\right] \mathrm{FDG}$ employs high performance anion exchange chromatography with pulsed amperometric detection (HPAEC-PAD) and radiometric detection. ${ }^{22-24}$ This strategy enables the separation of $\left[{ }^{18} \mathrm{~F}\right] \mathrm{FDG}$ from its 
carbohydrate byproducts, 2-chloro-2-deoxy-D-glucose (CIDG) and $\left[{ }^{18} \mathrm{~F}\right] 2$-fluoro-2-deoxy-D-mannose $\left(\left[{ }^{18} \mathrm{~F}\right] \mathrm{FDM}\right)$, and their quantification. A basic $(\mathrm{pH}>11)$ mobile phase (typically sodium hydroxide) renders the carbohydrates negatively charged, allowing both their separation on the strong anion exchange (SAX) column and their detection by PAD. A radiometric detector is used in conjunction with PAD in order to obtain radiation signals for the radiochemical identification of $\left[{ }^{18} \mathrm{~F}\right] \mathrm{FDG}$ based on the comparison of retention times with a $\left[{ }^{19}\right.$ F]FDG reference standard.

We are developing an integrated microfluidic platform for the $\mathrm{QC}$ analysis of dose-on-demand radiotracers, ${ }^{12,25-28}$ one component of which is intended to be a HPAEC-PAD module for the analysis of $\left[{ }^{18} \mathrm{~F}\right] \mathrm{FDG}$ and other carbohydrate-based radiotracers. ${ }^{29}$ This technique is expected to feature a monolithic HPLC column ${ }^{30-38}$ for the SAX separation since silica monoliths can be generated in microfluidic channels ${ }^{39}$ via the sol-gel method, and both our group ${ }^{27,40}$ and others ${ }^{41-44}$ have employed them for radiochemical processes. We have previously demonstrated several potential radiometric detection methods that could be applied to the module, ${ }^{27,28,45}$ including one in combination with a monolith. ${ }^{27}$ Here, we focus on the development of a pulsed amperometric detector (PAD) for the HPAEC-PAD module, using D-glucose as a generic proxy for carbohydrate-based radiotracers.

Two strategies for a PAD setup were investigated, each employing a gold working electrode (WE) with a sodium hydroxide mobile phase in order for catalytic oxidation of glucose to occur at the gold electrode surface, thus providing a signal. The first device utilised commercial, disposable screenprinted electrodes (SPES) in a polymer chip that built upon a previous design used for studying glutathione metabolites, ${ }^{46}$ and was designed towards a single-use cartridge as part of a larger integrated QC platform. The second device featured bulk wire electrodes in a glass chip, which also built on a previous platform used to measure enzyme inhibition ${ }^{47}$ and enzyme kinetics, ${ }^{48}$ and was designed to form a permanent fixture in a QC system rather than being a disposable cartridge, akin to a traditional HPAEC-PAD setup used in radiotracer QC. Both devices were applied to PAD here for the first time in a series of proof-of-concept experiments to determine their potential for carbohydrate-based radiotracer analysis. Initial testing of the SPE-based device also included cyclic voltammetry (CV) of ferricyanide on a carbon WE, before swapping to a gold WE for glucose analysis. D-Glucose solutions were introduced into each of the devices using a flow injection analysis (FIA)-style setup in order to mimic a HPAEC injection minus the SAX separation column, and the resultant $P A D$ responses were monitored to assess the viability of the strategies towards on-chip QC analysis of $\left[{ }^{18} \mathrm{~F}\right]$ FDG and other carbohydrate-based radiotracers.

\section{Experimental}

Chemicals and solutions
Sodium hydroxide $(\mathrm{NaOH})$, potassium chloride $(\mathrm{KCl})$, and $\mathrm{D}$ glucose were purchased from Sigma-Aldrich (Dorset, UK). Potassium ferricyanide $\left(\mathrm{K}_{3}\left[\mathrm{Fe}(\mathrm{CN})_{6}\right]\right)$ was purchased from Fisher Scientific (Loughborough, UK).

Solutions were prepared in double-filtered $(0.05 \mu \mathrm{m}$ pore size) high purity water $\left(18.2 \mathrm{M} \Omega \mathrm{cm}\right.$ at $\left.25^{\circ} \mathrm{C}\right)$ via an ELGA Option 4 system that fed into an ELGA UHG PS system, both from ELGA Process Water (Marlow, UK). A background electrolyte solution of $0.1 \mathrm{M} \mathrm{KCl}$ was prepared for carbon working electrode-based experiments. A $5 \mathrm{mM}$ potassium ferricyanide solution was prepared in $0.1 \mathrm{M} \mathrm{KCl}$ and further diluted with $0.1 \mathrm{M} \mathrm{KCl}$ to give a concentration range of 1 to 5 $\mathrm{mM}$. A mobile phase of $0.1 \mathrm{M} \mathrm{NaOH}$ was prepared for the PAD analysis of D-glucose. Electrolyte solutions were sonicated (without vacuum) for $20 \mathrm{~min}$ to remove some of the dissolved air, since dissolved oxygen can affect sensitivity, reproducibility, and baseline noise. ${ }^{49}$ However, with no vacuum applied, some oxygen will still be present in the electrolyte solutions. A stock solution of 1000 ppm D-glucose was prepared in purified water and further diluted in water to give a concentration range of 100 to $1000 \mathrm{ppm}$.
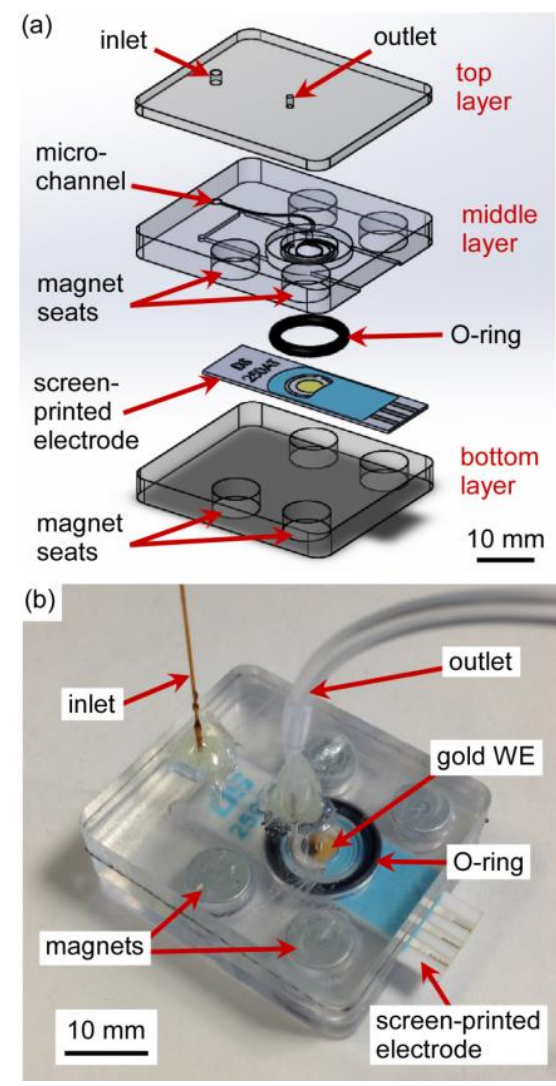

Fig. 1 Design and setup of the polymer microfluidic "SPE-chip" used for pulsed amperometric detection (PAD) with a commercial DropSens screen-printed electrode (SPE). (a) Exploded schematic showing the setup of the SPE-chip, which utilised an O-ring to seal the chip against the SPE. (b) Photograph of the assembled SPE-chip with inlet and outlet tubing and the use of magnets to fasten the device together around the SPE. 


\section{Fabrication and setup of screen-printed electrode chip}

The microfluidic device employed here for use with SPEs, referred to here as the "SPE-chip", was adapted from a previous version of the device used by Vasiliadou et al. ${ }^{46}$ but comprised a spiral channel around the electrode area rather than an open chamber as in the original design, together with a more convenient clamping system. The design for the SPEchip was prepared in SolidWorks (Dassault Systèmes SolidWorks Corporation, France) and milled into polycarbonate sheets via a computer numerically controlled (CNC) micromilling machine (Datron M7, Datron, Germany). ${ }^{50}$

The chip comprised three polycarbonate layers (Fig. 1). The top layer $(2 \mathrm{~mm}$ thick) featured only inlet $(400 \mu \mathrm{m} \varnothing)$ and outlet $(400 \mu \mathrm{m} \varnothing)$ access holes for tubing that fed into a microfluidic channel in the middle layer $(5 \mathrm{~mm})$. The underside of the middle layer contained a rectangular cutout that allowed an SPE to be seated in place. The underside further contained a seat for a O-ring that was used to seal the microfluidic channel against the SPE.

The inlet channel (100 $\mu \mathrm{m}$ wide by $20 \mu \mathrm{m}$ deep) was milled into the topside of the middle layer before passing through the thickness of the layer to the underside, above the electrode region of the SPE, allowing the fluid to be routed from the inlet hole to the electrode in a wall-jet mode. Above the electrode, the inlet channel formed a spiral that then directed the liquid around the working electrode and out of an outlet channel, which also passed through the thickness of the middle layer to the outlet hole. This alteration was intended to allow greater control of the flow through the device compared to the previous design of the device that comprised an open chamber around the electrode area. ${ }^{46}$ The top layer and the middle layer were aligned and bonded together using double-sided tape. The bottom layer consisted of a flat piece of polycarbonate ( $5 \mathrm{~mm}$ thick) on which the SPE was located.

While the original design of the chip had required the multiple layers to be glued together to seal the SPE as a semipermanent fixture, ${ }^{46}$ a strategy also adopted by other groups using glue ${ }^{51}$ or double-sided adhesive films, ${ }^{52}$ this led to long chip preparation times every time the SPE had to be replaced. Screw-based clamps have also been used by other groups to assemble a similar platform that allow faster SPE replacement, ${ }^{53}$ but magnets were employed here to allow the exchange of SPEs within a matter of seconds, drastically reducing downtime between experiments. A similar mechanism is also adopted by Metrohm DropSens's DRPFLWCL Flow-Cell for use with their SPEs in larger volume walljet mode electrochemical analysis in flow. Here, four circular magnet seats were milled into the topside of the bottom layer and the underside of the middle layer, and powerful neodymium-iron-boron (NdFeB) magnets $(8 \mathrm{~mm} \varnothing \times 4 \mathrm{~mm}$, Magnet Sales, Wiltshire, UK) were glued into each seat with Araldite Rapid epoxy resin (RS Components). When the two parts (the combined top/middle layers and the bottom layer) were subsequently brought together with an SPE and an O-ring between them, the attractive force of the magnets was sufficient to hold the assembly together without leakage. We note that Metrohm DropSens's DRP-FLWCL Flow-Cell operates in a somewhat similar fashion for larger volume wall-jet mode electrochemical analysis.

Metrohm Dropsens SPEs (Metrohm UK Ltd., Cheshire, UK) were used in conjunction with the SPE-chip for electrochemical analyses. Tests of the microfluidic device using cyclic voltammetry (CV) were performed using potassium ferricyanide in $0.1 \mathrm{M} \mathrm{KCl}$ as an analyte. These tests utilised Metrohm DropSens DRP-110 screen-printed electrodes (34 $\mathrm{mm} \times 10 \mathrm{~mm} \times 0.5 \mathrm{~mm}$ ) with a carbon working electrode (WE; $4 \mathrm{~mm} \varnothing)$, a carbon counter electrode (CE), and a silver reference electrode (RE) (see Fig. S1a in the ESI). PAD analysis of glucose was performed using Metrohm DropSens DRP250AT electrodes in the SPE-chip, which featured a gold WE (4 $\mathrm{mm} \varnothing$ ), platinum CE, and silver RE (see Fig. S1b in the ESI). The SPEs were linked to a potentiostat using a Metrohm DropSens DRP-CAST cable connector (see Fig. S1c in the ESI). The potentiostat was a PalmSens ${ }^{3}$ with PSTrace 4.8 software (PalmSens BV, Utrecht, Netherlands) that was purchased from Alvatek Ltd. (Romsey, Hampshire, UK).

Fused silica capillary (150 $\mu \mathrm{m}$ i.d. (inner diameter), $363 \mu \mathrm{m}$ o.d. (outer diameter), Polymicro Technologies, CM Scientific, UK) was glued into the inlet hole of the SPE-chip using Araldite Rapid epoxy resin in order to pump liquid into the chip. A short section of fused silica capillary was also glued into the outlet hole of the chip, to which a short length of PTFE tubing $(0.3$
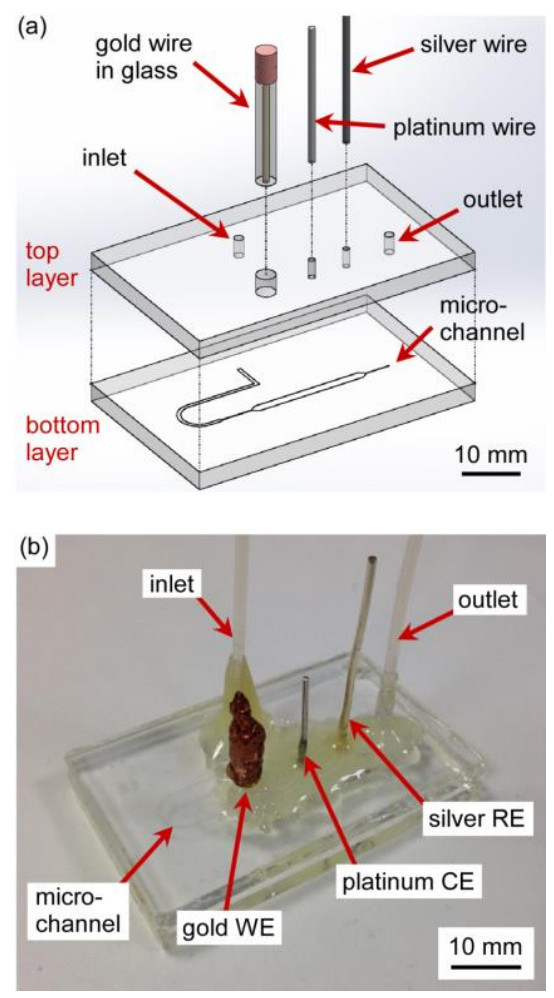

Fig. 2 Design and setup of the glass microfluidic "wire-chip" used for pulsed amperometric detection (PAD) with wire electrodes. (a) Exploded schematic showing the setup of the wire-chip and its electrodes. (b) Photograph of the assembled wire-chip with the wire electrodes incorporated. 
$\mathrm{mm}$ i.d., $1.8 \mathrm{~mm}$ o.d., Supelco, Sigma-Aldrich) was attached and finally a length of Tygon tubing $(1 \mathrm{~mm}$ i.d., $1.8 \mathrm{~mm}$ o.d., Cole-Parmer, UK) that directed the waste to a waste vial. Only one SPE-carrying microflow cell was tested, although preliminary tests were performed using the previous iteration of the device. ${ }^{46}$ A new SPE was used for every new series of experiments.

\section{Fabrication and setup of wire electrode chip}

The microfluidic chip device employed here for use with wire electrodes, referred to hereafter as the "wire-chip", was based on the device used previously by He et al. ${ }^{47,48}$ The design was prepared using AutoCAD software (Autodesk, Inc.) from which a photomask was produced by JD Photo Data (Hitchin, Hertfordshire, UK). The chip was fabricated in $1 \mathrm{~mm}$ thick B270 glass (Telic, CA, USA) from the photomask using standard photolithography and wet etching methods. ${ }^{54}$ The final depth of the channel structure was $50 \mu \mathrm{m}$.

The chip design featured several channel sections in series (Fig. 2a). The inlet channel was $20 \mathrm{~mm}$ long and $600 \mu \mathrm{m}$ wide; while it had been used in previous work to contain a sol-gel silica monolith, it was left empty here. A short channel linking the inlet channel to the electrochemical channel was $5 \mathrm{~mm}$ long and $100 \mu \mathrm{m}$ wide, and the electrochemical detection channel was $20 \mathrm{~mm}$ long and $1.5 \mathrm{~mm}$ wide. The final outlet channel was $4 \mathrm{~mm}$ long and $100 \mu \mathrm{m}$ wide.

The etched glass plate was thermally bonded to a $3 \mathrm{~mm}$ thick B270 glass plate into which access holes had been drilled: for the inlet and outlet holes $(1.5 \mathrm{~mm} \varnothing)$, the working electrode $(3 \mathrm{~mm} \varnothing)$, and the counter and reference electrodes (1 $\mathrm{mm} \varnothing$ ). PTFE tubing ( $0.3 \mathrm{~mm}$ i.d., $1.8 \mathrm{~mm}$ o.d.) was inserted into the inlet and outlet holes and glued into place with epoxy resin (Fig. 2b).

A gold working electrode was fabricated by sealing a $1 \mathrm{~mm}$ $\varnothing$ gold wire (Goodfellow Cambridge Ltd., Huntingdon, UK) sealed inside a glass body $(3 \mathrm{~mm} \varnothing)$ and connecting the top of the wire to copper foil tape (35 $\mu \mathrm{m}$ thick, AT525, Advance Tapes International Ltd., Leicester, UK) that was wrapped around the top of glass body (see Fig. S2a in the ESI). The opposite end of the gold wire was flush with the bottom of the glass body, thus acting as a $1 \mathrm{~mm} \varnothing$ disc electrode. The original wire-chip platform ${ }^{47,48}$ employed a platinum wire working electrode $(0.5 \mathrm{~mm} \varnothing)$ that was easy to incorporate within a glass body during lampworking (at $\sim 1,000-1,200{ }^{\circ} \mathrm{C}$ ) due to platinum's high melting point $\left(1,768^{\circ} \mathrm{C}\right) .{ }^{55}$ The lower melting point of gold $\left(1,064{ }^{\circ} \mathrm{C}\right)^{55}$ meant that more care had to be taken when sealing the wire within the glass, hence a thicker wire was used in order to maintain the wire's integrity during the electrode fabrication process.

The counter and reference electrodes consisted of a platinum wire ( $1 \mathrm{~mm} \varnothing$, Goodfellow), and a silver wire ( $1 \mathrm{~mm}$ $\varnothing$, Goodfellow), respectively. The electrodes were polished with alumina paste to clean the surfaces before being inserted into the relevant access holes and glued in place with epoxy resin. The electrodes were inserted such that the liquid flowing through the chip encountered the gold WE first, followed by the platinum $\mathrm{CE}$, and finally the silver RE. The electrodes were connected to the PalmSens ${ }^{3}$ potentiostat using a Metrohm DropSens DRP-CAC cable connector with crocodile clips (see Fig. $S 2 b$ in the ESI).

\section{Cyclic voltammetry (CV) of ferricyanide in the SPE-chip}

The SPE-chip was tested by performing CV of the ferrocyanide/ferricyanide redox couple on the carbon WEbased DRP-110 SPE. The SPE-chip was placed inside a Faraday cage was and washed with $1 \mathrm{~mL}$ of $0.1 \mathrm{M} \mathrm{KCl}$ solution, followed by the injection of $0.2 \mathrm{~mL}$ of potassium ferricyanide ( $5 \mathrm{mM}$ in $0.1 \mathrm{M} \mathrm{KCl}$ ) into the chip. CV was then performed on the ferricyanide between $-0.9 \mathrm{~V}$ and $+0.9 \mathrm{~V}$, at a scan rate of $0.09 \mathrm{~V} \mathrm{~s}^{-1}$. The effect of changing the $E_{\text {step }}$ value (i.e. the incremental step in potential during the scan) was studied between 0.001-0.2 V, and the response to varying ferricyanide concentrations (0-5 $\mathrm{mM}$ ) was studied. The effect of flow rate was also studied by pumping $5 \mathrm{mM}$ potassium ferricyanide through the SPE-chip using a Harvard Apparatus 11 Plus syringe pump (Biochrom Ltd., Cambourne, UK) at flow rates of 100-1000 $\mu \mathrm{L} \mathrm{h}^{-1}\left(1.7-16.7 \mu \mathrm{L} \mathrm{min}^{-1}\right)$ during CV experiments.

\section{Flow injection analysis (FIA) setup}

An FIA setup was employed to inject aliquots of D-glucose into a stream of $0.1 \mathrm{M} \mathrm{NaOH}$ mobile phase that was continuously pumped into either the SPE-chip (containing a DRP-250AT electrode with a gold WE) or the wire-chip (featuring the gold wire WE) in order to perform PAD. The selected chip was placed within a Faraday cage and connected to a PalmSens ${ }^{3}$ potentiostat, as described above, located outside the cage (see Fig. S3 in the ESI). A new SPE was used for each experiment.

The inlet tubing of the chip was connected to a Diba Omnifit ${ }^{\circledR}$ Labware Low Pressure 6-Port Loop Manual Sample Injection Valve (Diba Industries Inc.), purchased from Kinesis Ltd. (Cole-Parmer, Cambridgeshire, UK), that was positioned outside of the cage. A Harvard Apparatus 11 Plus syringe pump was used to pump $0.1 \mathrm{M} \mathrm{NaOH}$ mobile phase from a $10 \mathrm{~mL}$ disposable plastic syringe (BD Plastipak, VWR, Lutterworth, UK) through the chip via the 6-port injection valve. A sample loop was fabricated from a $6 \mathrm{~cm}$ length of fused silica capillary (150 $\mu \mathrm{m}$ i.d., $363 \mu \mathrm{m}$ o.d.), which stored $1 \mu \mathrm{L}$ of $\mathrm{D}$-glucose sample solution that was introduced via a $1 \mathrm{~mL}$ disposable plastic syringe (BD Plastipak, VWR) into the sample injection port on the valve when in the "Load" position. When the 6-port valve was then turned to the "Inject" position, the $1 \mu \mathrm{L}$ of stored sample was injected into the mobile phase and passed through the microfluidic chip for PAD analysis. The PSTrace 4.8 detection software was operated in multiple pulse amperometry (MPAD) mode and used to store the resultant amperograms for each D-glucose injection.

\section{Results and Discussion}

\section{Cyclic voltammetry (CV) of ferricyanide in the SPE-chip}

The SPE-chip and the use of the SPEs were tested by performing $\mathrm{CVs}$ on the ferrocyanide/ferricyanide redox couple 
using the carbon WE-based DRP-110 SPE. In the forward scan of this redox couple, ferrocyanide $\left(\mathrm{Fe}^{\prime \prime}(\mathrm{CN})_{6}{ }^{4-}\right)$ is oxidised to ferricyanide $\left(\mathrm{Fe}^{\text {III }}(\mathrm{CN})_{6}{ }^{3-}\right)::^{56,57}$

$\mathrm{Fe}^{\prime \prime}(\mathrm{CN})_{6}^{4-} \rightarrow \mathrm{Fe}^{\mathrm{II \prime}}(\mathrm{CN})_{6}{ }^{3-}+\mathrm{e}^{-}$

In the reverse scan, ferricyanide $\left(\mathrm{Fe}^{\mathrm{III}}(\mathrm{CN})_{6}{ }^{3-}\right)$ is reduced to ferrocyanide $\left(\mathrm{Fe}^{\prime \prime}(\mathrm{CN})_{6}{ }^{4-}\right)$ :

$\mathrm{Fe}^{\prime \prime \prime}(\mathrm{CN})_{6}^{3-}+\mathrm{e}^{-} \rightarrow \mathrm{Fe}^{\prime \prime}(\mathrm{CN})_{6}^{4-}$

$\mathrm{CV}$ experiments were performed using $5 \mathrm{mM}$ potassium ferricyanide in $0.1 \mathrm{M} \mathrm{KCl}$ and the effect of varying several of the parameters was observed.

CV scans were performed between $+0.9 \mathrm{~V}$ and $-0.9 \mathrm{~V}$, and an example CV is shown in Fig. S4 of the ESI using a scan rate of $0.09 \mathrm{~V} \mathrm{~s}^{-1}$ and an $E_{\text {step }}$ of $0.005 \mathrm{~V}$. The voltammogram yielded an anodic peak current $\left(i_{\mathrm{pa}}\right)$ for the oxidation of ferrocyanide to ferricyanide at $\sim+0.19 \mathrm{~V}$, and a cathodic peak current $\left(\mathrm{i}_{\mathrm{pc}}\right)$ for the reduction of ferricyanide to ferrocyanide at $\sim+0.05 \mathrm{~V}$. The effect of changing the $E_{\text {step }}$ value was observed by varying it between 0.001 and $0.2 \mathrm{~V}$ at a scan rate of $0.09 \mathrm{~V} \mathrm{~s}^{-1}$. The peak currents largely remained unchanged (see Fig. S5 in the ESI) unless using the highest or lowest $E_{\text {step }}$ values, hence an $E_{\text {step }}$ of $0.009 \mathrm{~V}$ was used for subsequent experiments.

CVs were then performed on a range of potassium ferricyanide concentrations between 0-5 $\mathrm{mM}$ to examine the possibility of achieving quantitative analysis with the SPE-chip (scan rate $=0.09 \mathrm{~V} \mathrm{~s}^{-1}, \mathrm{E}_{\text {step }}=0.009 \mathrm{~V}$ ). The resultant $\mathrm{CV}$ s are shown in Fig. S6a in the ESI, and calibration curves were successfully constructed using the peak anodic currents $\left(i_{p a}\right)$ and cathodic currents $\left(i_{p c}\right)$ that showed good linearity (see Fig. $\mathrm{S} 6 \mathrm{~b}$ in the ESI), demonstrating the potential of the device for quantitative analysis.

Finally, the effect of performing analyses in a flowing system was tested to ensure that the device was suitable for HPAEC and similar separation mechanisms. $5 \mathrm{mM}$ potassium ferricyanide was pumped into the SPE-chip using a syringe pump at flow rates of 100-1000 $\mu \mathrm{L} \mathrm{h}^{-1}\left(1.7-16.7 \mu \mathrm{L} \mathrm{min}^{-1}\right)$, and $\mathrm{CV}$ was performed on the flowing solution (scan rate $0.09 \mathrm{~V} \mathrm{~s}^{-1}$, $E_{\text {step }}=0.009 \mathrm{~V}$ ). The CVs are shown in Fig. S7a in the ESI, and demonstrated that performing in-flow analysis with the SPEchip was feasible. The peak current for the reduction of ferricyanide was plotted against the cube root of the flow rate (see Fig. S7b in the ESI), demonstrating a linear relationship that indicated the mass transport occurred predominantly by convection rather than diffusion in the flowing system. ${ }^{58-60}$ It is worth noting that the flow rate dependence of the peak current means that calibration curves of reagent concentration only apply to a given flow rate. ${ }^{59}$

\section{Cyclic voltammetry (CV) of D-glucose in the SPE-chip}

The analysis of D-glucose was performed using PAD as a substitute for carbohydrate-based radiotracers and byproducts, such as FDG and CIDG, that would be the target analytes in a future version of the integrated microfluidic $\mathrm{QC}$ platform. Prior to performing PAD analysis of D-glucose, CV was performed using the DRP-250AT SPE with a gold WE in the SPE-chip to observe the signal to determine the characteristics of the response.

A $1000 \mathrm{ppm}$ solution of D-glucose was scanned in the range of $-1.00 \mathrm{~V}$ to $+0.75 \mathrm{~V}$ (scan rate $=0.02 \mathrm{~V} \mathrm{~s}^{-1}, \mathrm{E}_{\text {step }}=0.001$ $\mathrm{V})$, alongside a $0.1 \mathrm{M} \mathrm{NaOH}$ solution used as the background electrolyte, and the voltammograms are shown in Fig. 3 . As the voltage is increased in the positive direction, the catalytic oxidation of the glucose molecule occurs at the gold electrode surface in the alkaline medium, producing a current that is detected as a signal. ${ }^{61-63}$ The oxidation peaks at about $+0.6 \mathrm{~V}$, which is also the point at which gold oxide starts to form on the electrode surface. As the voltage is further increased above $+0.6 \mathrm{~V}$, the current drops since the formation of the gold oxide inhibits the oxidation of glucose. Thus, whilst the current peak occurs at about +0.6 V, a lower detection potential should be used so as to limit the effects of gold oxidation on the electrode that affects the signal-to-noise ratio. ${ }^{61}$ As the scan is then reversed, the current becomes negative as the gold oxide is reduced, thus regenerating the electrode surface back to gold.

The scan of the $0.1 \mathrm{M} \mathrm{NaOH}$ electrolyte solution notably lacks the characteristic glucose oxidation peak on the forward (positive voltage) scan, indicating the region in which a glucose signal can be detected against the electrolyte background. The increase in current at high voltage may indicate the evolution of oxygen from the electrolyte solution, whilst the large decrease in current at low voltage may indicate oxygen reduction, both likely due to incomplete degassing of the electrolyte solution.

The CV provided a starting point for choosing the voltages to be used in the PAD of D-glucose.

\section{Pulsed amperometric detection using the SPE-chip}

While the detection of glucose in an alkaline medium occurs by

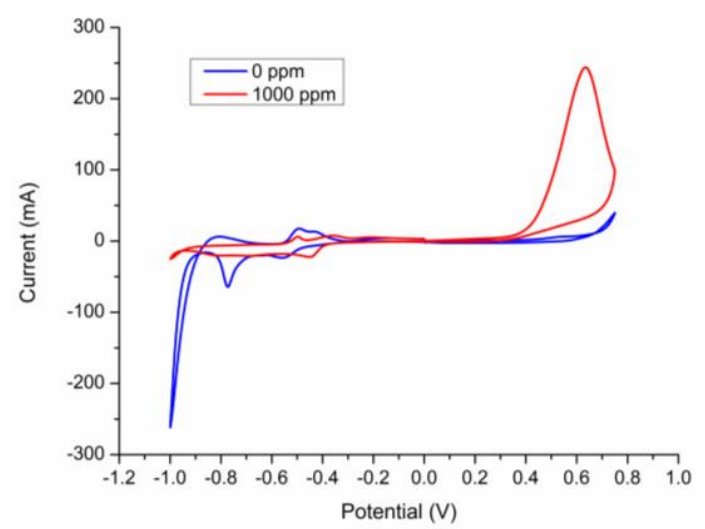

Fig. 3 Cyclic voltammograms (CVs) of D-glucose in $0.1 \mathrm{M} \mathrm{NaOH}$, obtained using a screen-printed electrode (SPE) with a gold working electrode in the SPE-chip. CVs were performed on fresh electrodes from $0 \mathrm{~V}$ to $+0.75 \mathrm{~V}$ (oxidation), then to -1.00 $\mathrm{V}$ (reduction), and back to $0 \mathrm{~V}$. 
oxidation at the gold electrode surface using a positive potential, the oxidation products poison the electrode and inhibit further analyte oxidation. ${ }^{61,64-67}$ This necessitates a triple-pulse waveform pulse (see Fig. 4a for an illustration) in order to perform amperometric detection. Here, the waveform not only supplies this first potential to take a measurement ( $E 1$, measurement step), but then applies a further two potentials (E2 and E3) in order to clean the electrode in preparation for further measurements. The second potential is much higher than E1 and oxidatively cleans the gold electrode of the poisons (E2, electrode cleaning step). The third potential has a negative value, which reduces the oxide layer formed by E2 back to gold (E3, electrode regeneration step), thus enabling further detection on the cleaned gold surface. One issue with this particular type of triple-pulse waveform, however, is that wearing of the working electrode occurs over time and thus causes a gradual decrease in signal.

A triple-potential PAD waveform was employed here for the detection of D-glucose samples injected into SPE-chip via the FIA system at $10 \mu \mathrm{L} \mathrm{min}^{-1}$. The SPE was a DRP-250AT with a gold WE. The PAD potentials employed are shown in Table 1 , and were selected based on the CV data, a study of the effect of varying each potential (see Fig. S8 of the ESI), and from values found in the literature. ${ }^{23,24,66,68-72}$ A further effect that informed the potentials was the extent of wearing of the gold WE, with high E1 values, for example, leading to rapid fouling of the electrode (see Fig. S9 in the ESI). Hence, potentials were kept relatively low to maintain electrode efficiency for longer, despite the gains in sensitivity that could be achieved by employing higher E1 potentials (see Fig. S8a in the ESI for example). The fouling patterns on the gold electrodes also demonstrated that the solution passing through chip followed the spiral design of the microchannel structure.

Table 1. PAD settings for the detection of glucose in $0.1 \mathrm{M}$ $\mathrm{NaOH}$ at a flow rate of $10 \mu \mathrm{L} \mathrm{min}^{-1}$ during FIA injections.

\begin{tabular}{|c|c|c|}
\hline Step & Potential (V) & Time (s) \\
\hline E1 & +0.30 & 0.44 \\
\hline E2 & +0.80 & 0.18 \\
\hline E3 & -0.30 & 0.36 \\
\hline
\end{tabular}

Having established that the SPE-chip with a gold WE could be used to detect D-glucose via PAD in an FIA setup, its potential for performing HPAEC-PAD of FDG was examined by performing a glucose calibration. D-glucose standards having concentrations of $0,100,250,500,750$, and $1000 \mathrm{ppm}$ in 0.1 $\mathrm{M} \mathrm{NaOH}$ were injected multiple times into the SPE-chip at a flow rate of $10 \mu \mathrm{L} \mathrm{min}^{-1}$ and the signal peaks were detected by PAD. Typical signal peaks from each glucose concentration are shown overlaid on top of each other in Fig. $4 \mathrm{~b}$ and demonstrate the increase in peak height and peak area with increasing concentration. The FIA setup suffered from some issues with sample injection reproducibility, resulting in the occasional disparity in peak sizes, and the sporadic introduction of air bubbles into the system. The presence of dissolved oxygen due to the incomplete degassing process of the electrolyte solution may also have affected the reproducibility.

A calibration curve was constructed from the amperograms and is shown in Fig. 4c, based on the peak area of each glucose signal. From the slope $(S)$ of the calibration curve and the standard deviation $(s)$ of the blank measurements, the limit of detection (LOD) of the platform with its current parameters (Table 1 ) was calculated using the equation LOD $=3.3 \mathrm{~s} / \mathrm{S}$ to be $0.1 \mathrm{ppm}(0.6 \mu \mathrm{M}) .^{73}$ The limit of quantification (LOQ) was calculated using the equation $\mathrm{LOQ}=10 \mathrm{~s} / \mathrm{S}$ to be $0.3 \mathrm{ppm}(1.7$ $\mu \mathrm{M})$.

An LOD of $0.1 \mathrm{ppm}$ puts it on a par with the handful of other microfluidic PAD devices employed for glucose analysis, which typically exhibited LODs of around 4 to $16 \mathrm{ppm}$ (20 to 90 $\mu \mathrm{M}){ }^{74-76}$ down to $0.2 \mathrm{ppm}(1.2 \mu \mathrm{M}) .^{77}$ The SPE-chip performance also compares favourably with some examples of liquid chromatography using commercial PAD detectors, ${ }^{72,78}$ but currently cannot reach the limits that can be achieved with such detectors, e.g. $14 \mathrm{ppb}(80 \mathrm{nM})$ by LaCourse et al. ${ }^{71,79}$ and 5 ppb (28 nM) by Antec Scientific. ${ }^{80}$

When HPAEC-PAD is employed for the analysis of FDG and CIDG in the QC of $\left[{ }^{18} \mathrm{~F}\right] \mathrm{FDG}$, the concentration threshold for each is $0.5 \mathrm{mg} / V$, where $V$ is the maximum recommended dose in millilitres. For example, if a dose of $\left[{ }^{18} \mathrm{~F}\right] \mathrm{FDG}$ is prepared in $20 \mathrm{~mL}$ then the threshold for FDG and CIDG is $25 \mathrm{ppm}$; for a 10 $\mathrm{mL}$ dose it is $50 \mathrm{ppm}$. The ABT BG-75 Biomarker Generator ${ }^{7,8,81}$ is a compact cyclotron platform, with an integrated radiochemistry module, that produces $\left[{ }^{18} \mathrm{~F}\right] \mathrm{FDG}$ using a doseon-demand compatible method. This technology is available in the PET Research Centre at the University of Hull and generates a dose volume of $\sim 2.2 \mathrm{~mL}$, hence the threshold for FDG and CIDG is $227 \mathrm{ppm}$. Therefore, even if the sensitivity to FDG or CIDG is somewhat less than the $0.1 \mathrm{ppm}$ LOD for Dglucose, we anticipate that the SPE-chip should be more than capable of detecting relevant concentrations of FDG and CIDG. Furthermore, the sensitivity of the SPE-chip method could be further improved by optimising the PAD waveform; the effect of varying each potential was examined individually (see Fig. S8 in the ESI), but the effect of changing all three parameters at the same time has not been investigated. Doing so will enable an optimised waveform to be established, which will enhance the sensitivity and longevity of the SPE. In general, the optimisation of PAD is more challenging than for other electrochemical techniques due to the number of parameters that can be adjusted. ${ }^{82}$

While non-radioactive forms of FDG and CIDG were not tested here, some estimations of the ability of the SPE-chip to detect them can be made based on FDG detection in the literature. For example, Antec Scientific demonstrated the use of their HPAEC-PAD system for the separation and analysis of D-glucose, FDG, CIDG and FDM, obtaining an LOD for glucose (5 ppb) that was 7.2 times higher for FDG, 6 times higher for CIDG, and 17 times higher for FDM. ${ }^{80}$ Assuming that similar differences in LOD occur using the SPE-chip, based on a 0.1 ppm LOD for glucose, the LODs would thus be $0.7 \mathrm{ppm}$ for FDG, 0.6 ppm for CIDG, and 1.7 ppm for FDM, thus still being 
well below the threshold required for FDG quality control These are estimations and such performance would need to be tested in the future, but it nonetheless shows the promise of the platform.

The results demonstrate that the use of commercial SPEs in a microfluidic flow cell is a viable option for performing PAD in an integrated $\left[{ }^{18}\right.$ F]FDG QC platform. The SPEs are designed to be disposable, which fits well with one potential design ethos for the QC platform wherein the system, including the HPAEC-

(a)

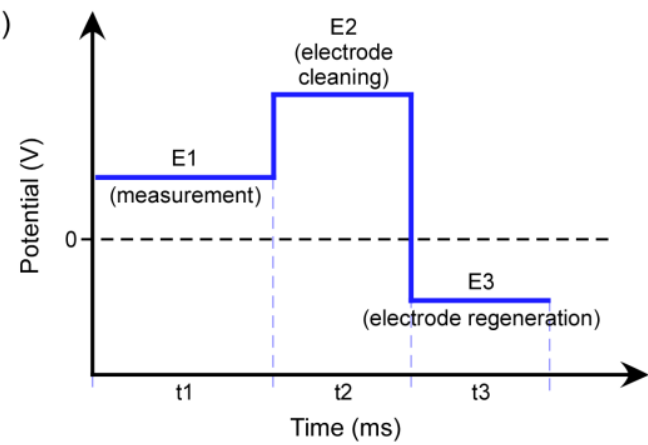

(b)
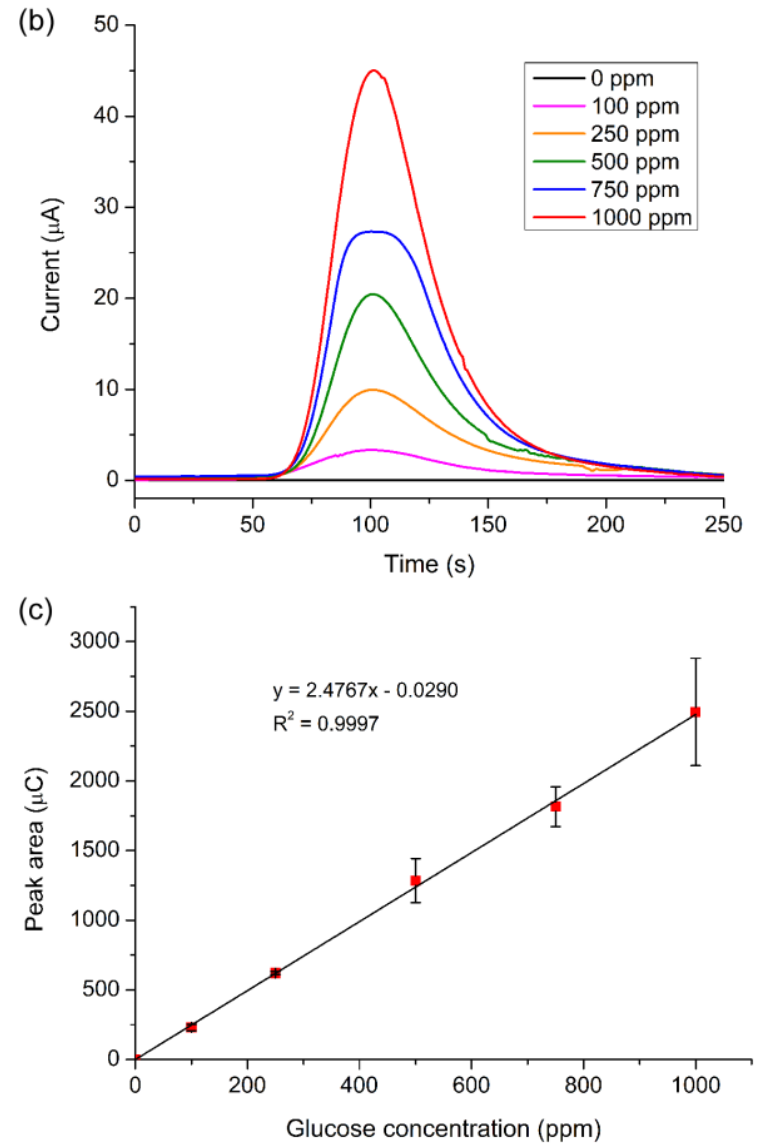

Fig. 4 Pulsed amperometric detection (PAD) of D-glucose in 0.1 $\mathrm{M} \mathrm{NaOH}$ using the SPE-chip with a gold working electrode. (a) Schematic representation of the triple-potential waveform of a PAD pulse for detection of glucose and subsequent cleaning of the electrode. (b) Amperograms showing a single, representative flow injection analysis (FIA) signal peak detected via PAD for varying glucose concentrations. Greater concentrations yielded greater peak heights and peak areas. (c) Calibration curve for the detection of glucose in the SPEchip.
PAD component utilising a monolithic anion exchange column, would comprise a single-use cartridge. This is the preferred method of operation to ensure compliance with good manufacturing practice (GMP) production standards for the PET radiotracer in an integrated dose-on-demand QC device. In this scenario, the fouling of electrodes would be of lesser concern since only a limited number of measurements would be required, thus allowing higher potentials to be applied that could improve sensitivity, although the fouling could also be avoided by optimisation of the triple-potential waveform. Further improvements to the design and assembly of the SPEchip are also envisaged to optimise the flow through the system.

\section{Pulsed amperometric detection using the wire-chip}

While one design ethos of the QC platform is to incorporate all of the analytical components into a single-use cartridge, another strategy would be to include most of the features onto the cartridge but have some be a more a permanent fixture of the overall apparatus. Here, sample would be injected into both the cartridge and the permanent components, either in series or in parallel, to achieve QC analysis. The HPAEC-PAD system could constitute one of those permanent fixtures, featuring an anion exchange separation column and a more robust electrochemical detection cell for longer term use than may be afforded by an SPE, i.e. the chip would take the role of a traditional HPLC system used in radiotracer $\mathrm{QC}$, acting as a permanent lab fixture.

The wire-chip is a glass device that features an electrochemical cell containing wire "bulk" electrodes that may be more suitable for longer term use. ${ }^{47,48}$ The device also contained a channel which has, in the previous work, ${ }^{47,48}$ housed a sol-gel silica monolith fabricated in situ for performing enzyme-immobilised reactions. This feature could potentially be exploited to form a silica monolith-based separation column in a QC platform, though this was not tested here. However, the electrochemical cell was tested for its potential for performing PAD analysis of D-glucose using a gold wire WE. The same triple-potential waveform was used as in the SPE-chip (see Table 1), as was the FIA setup for the injection of D-glucose standards.

Multiple injections of $0,100,250,500,750$, and 1000 ppm D-glucose in $0.1 \mathrm{M} \mathrm{NaOH}$ were performed and detected using the wire-chip. Fig. 5a shows an overlay of a typical single detection peak from each concentration for comparison, with increasing concentrations yielding greater peak heights and peak areas. The noise in the baseline is also quite high, which could be in part influenced by dissolved oxygen in the electrolyte solution. This could be improved in the future by the degassing of the mobile phase prior to or during HPAECPAD analyses.

A calibration curve was constructed from the signal peak areas for each D-glucose injection and is shown in Fig. $5 \mathrm{~b}$, from which an LOD of $9 \mathrm{ppm}(49 \mu \mathrm{M})$ and an LOQ of $27 \mathrm{ppm}(147$ $\mu \mathrm{M})$ were calculated for D-glucose as described previous. Although these are notably higher limits than were achieved using the SPE-chip, they are on a par with many other 

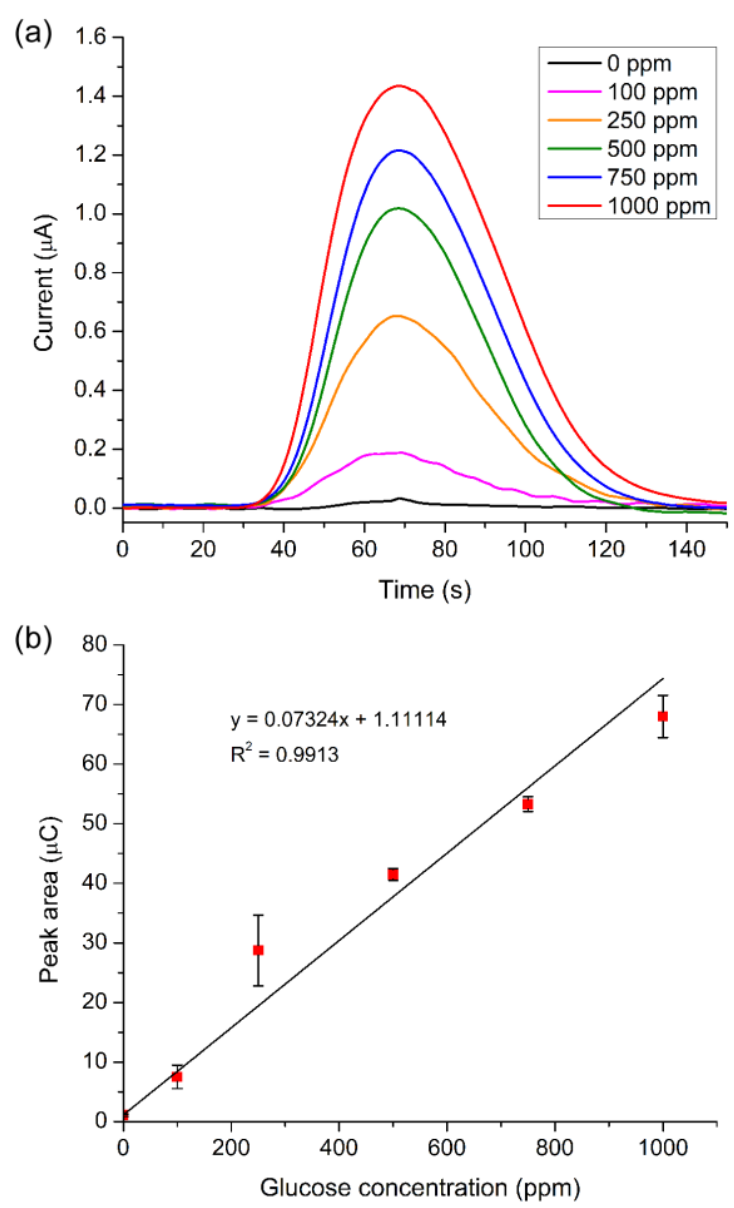

Fig. 5 Pulsed amperometric detection (PAD) of D-glucose in 0.1 $\mathrm{M} \mathrm{NaOH}$ using the wire-chip with a gold working electrode. (a) Amperograms showing a single representative detection peak from flow injection analysis (FIA) of varying concentrations of glucose. Greater concentrations of glucose yielded greater larger peak heights and peak areas. (b) Calibration curve of glucose obtained using the wire-chip.

microfluidic PAD examples. ${ }^{74-76}$ Like the SPE-chip, nonradioactive forms of FDG and CIDG were not tested here but the LODs for these molecules can be approximated using data from Antec Scientific, as in the previous section, yielding estimated LODs of 65 ppm for FDG, 54 ppm for CIDG, and 153 ppm for FDM. However, these values are approximations and the performance of the wire-chip would need to be assessed experimentally in the future.

The results indicate that the wire-chip, comprising a custom electrode setup, is not only capable of detecting glucose in a PAD format but may be also suitable for detection in the ranges suitable for $\left[{ }^{18} \mathrm{~F}\right]$ FDG QC analysis, particularly for lower volume doses that do not require low LODs. As with the SPE-chip, further optimisation of the PAD waveform, alongside the FIA injection system and the microfluidic design, would make the wire-chip a potentially viable option for an integrated QC platform.

\section{Conclusions}

We have developed and tested two on-chip methods of performing pulsed amperometric detection (PAD) of glucose, towards the quality control (QC) analysis of carbohydratebased radiotracers, such as $\left[{ }^{18} \mathrm{~F}\right] 2$-fluoro-2-deoxy-D-glucose $\left(\left[{ }^{18} \mathrm{~F}\right]\right.$ FDG $)$, via an integrated microfluidic QC platform. The first method utilised a disposable screen-printed electrode (SPE) incorporated into a microfluidic device that could be used as part of a single-use QC platform. The second method used wire electrodes that would be more suitable as a permanent, reusable analytical fixture.

In a series of proof-of-concept experiments, glucose standards were injected into each device in a flow injection analysis (FIA) format. The SPE-housing microfluidic flow cell achieved a limit of detection (LOD) of 0.1 ppm was using commercially available SPEs, whilst the wire-chip yielded an LOD of 9 ppm using in-house prepared wire electrodes. Each of the LODs were below the threshold limits required for $\left[{ }^{18} \mathrm{~F}\right] \mathrm{FDG}$ $\mathrm{QC}$ testing, particularly in low doses of $\left[{ }^{18} \mathrm{~F}\right] \mathrm{FDG}$, making both strategies potentially viable options for a miniaturised $Q C$ platform. However, further testing using non-radioactive FDG, CIDG and FDM will be required to confirm this, beyond which other carbohydrate-based tracers could be tested.

Future work will involve the optimisation of at least one of the devices in terms of the applied waveform and its integration with a separation channel in order to separate the target analytes in $\left[{ }^{18} \mathrm{~F}\right] \mathrm{FDG}$ doses prior to their measurement using PAD. The effects of changing each individual potential of the waveform has undergone some examination here but the relative effects of changing all three potentials will need to be tested further in order to obtain an optimum waveform. The degassing of the mobile phase either prior to or during (as in traditional HPLC systems) HPAEC-PAD analysis will also reduce noise and improve sensitivity and reproducibility whilst offering the usual benefits to HPLC analysis (e.g. less erratic flow rates). A more robust and accurate on-chip sample injection mechanism for introducing boluses of sample into the flowing mobile phase will also be required in future iterations. Optimisation of the flow through the SPE-chip by modifying the design and assembly is also envisaged to take it beyond the proof-of-concept stage. With such improvements in mind, a more complete series of tests are envisaged for confirming reproducibility once optimum conditions have been established.

These features will allow the PAD mechanism to be incorporated into either a high performance anion exchange chromatography system (HPAEC-PAD), potentially incorporating a sol-gel silica monolith as we have previously employed for radiopharmaceutical work. ${ }^{27,40}$ Alternatively, capillary electrophoresis (CE) could be employed for separation; CE and microfluidic CE have recently been applied towards the QC analysis of radiotracers, ${ }^{13,83,84}$ while microfluidic CE-PAD has already been shown as being suitable for carbohydrate analysis, ${ }^{75,76,85}$ thus making CE a potentially viable option. 


\section{Acknowledgements}

The authors thank the Royal Society of Chemistry (RSC) Analytical Chemistry Trust Fund (ACTF, grant no. ACSS 15/029), the Daisy Appeal charity (grant no. DAhul2011) and HEIF (University of Hull) for funding. The authors also thank $\mathrm{Dr}$ Assem Allam and his family for the generous donation to help found the PET Research Centre at the University of Hull and for their continued support. Julia Malle is thanked for the fabrication of the gold-wire-in-glass-body electrode. The reviewers are also thanked for their useful comments and suggestions that helped to improve the manuscript.

\section{Notes and references}

1. M. E. Phelps, Ann. Rev. Nucl. Part. Sci., 2002, 52, 303-338

2. P. H. Elsinga, Nucl. Med. Rev., 2012, 15, C13-C16.

3. P. Y. Keng, M. Esterby and R. M. Van Dam, "Emerging Technologies for Decentralized Production of PET Tracers", in Positron Emission Tomography - Current Clinical and Research Aspects, C.-H. Hsieh (Ed.), InTech, 2012, doi: 10.5772/31390.

4. V. Arima, G. Pascali, O. Lade, H. R. Kretschmer, I. Bernsdorf, V. Hammond, P. Watts, F. De Leonardis, M. D. Tarn, N. Pamme, B. Z. Cvetkovic, P. S. Dittrich, N. Vasovic, R. Duane, A. Jaksic, A. Zacheo, A. Zizzari, L. Marra, E. Perrone, P. A. Salvadori and R. Rinaldi, Lab Chip, 2013, 13, 2328-2336.

5. G. Pascali, G. Mazzone, G. Saccomanni, C. Manera and P. A. Salvadori, Nucl. Med. Biol., 2010, 37, 547-555.

6. G. Pascali, G. Nannavecchia, S. Pitzianti and P. A. Salvadori, Nucl. Med. Biol., 2011, 38, 637-644.

7. V. Awasthi, J. Watson, H. Gali, G. Matlock, A. McFarland, J. Bailey and A. Anzellotti, Appl. Radiat. Isot., 2014, 89, 167175.

8. A. Anzellotti, J. Bailey, D. Ferguson, A. McFarland, P. Bochev, G. Andreev, V. Awasthi and C. Brown-Proctor, J Radioanal Nucl Chem, 2015, 305, 387-401.

9. G. Pascali and L. Matesic, "How Far Are We from Dose On Demand of Short-Lived Radiopharmaceuticals?", in Perspectives on Nuclear Medicine for Molecular Diagnosis and Integrated Therapy, Y. Kuge, T. Shiga and N. Tamaki (Ed.), Springer Japan, Tokyo, 2016, pp. 79-92, doi: 10.1007/978-4-431-55894-1_6.

10. M. D. Tarn and N. Pamme, "Microfluidics", in Elsevier Reference Module in Chemistry, Molecular Sciences and Chemical Engineering, J. Reedijk (Ed.), Elsevier, Waltham, MA, 2013, doi: 10.1016/B978-0-12-409547-2.05351-8.

11. N. Ha, S. Sadeghi and R. van Dam, Micromachines, 2017, 8, 337.

12. M. D. Tarn, A. Isu, S. J. Archibald and N. Pamme, Proceedings of The 18th International Conference of Miniaturized Systems for Chemistry and Life Sciences (MicroTAS 2014), San Antonio, TX, USA, 2014.1077-1079.

13. J. Ly, N. S. Ha, S. Cheung and R. M. van Dam, Anal. Bioanal. Chem., 2018.

14. S. Yu, Biomed. Imag. Interv. J., 2006, 2, e57.

15. J. C. Hung, J. Nucl. Med., 2002, 43, 1495-1506.
16. G. Dietzel, J Radioanal Nucl Chem, 2003, 257, 187-189.

17. British Pharmacopoeia 2012, TSO, Norwich, 2012.

18. European Pharmacopoeia 8th Edition, EDQM Council of Europe, Strasbourg, 2013.

19. United States Pharmacopeia (37th Edition) and National Formulary (32nd Edition) (USP-NF), Rockville, 2014.

20. The International Pharmacopoeia Fourth Edition Phaemacopoea Internationalis Edito Quarta - Third Supplement, Geneva, 2013.

21. Chemistry, Manufacturing, and Controls (CMC), Silver Spring, 2011.

22. D. L. Alexoff, R. Casati, J. S. Fowler, A. P. Wolf, C. Shea, D. J. Schlyer and C.-Y. Shiue, Appl. Radiat. Isot., 1992, 43, 13131322.

23. C. Lemaire, P. Damhaut, B. Lauricella, C. Mosdzianowski, J. L. Morelle, M. Monclus, J. Van Naemen, E. Mulleneers, J. Aerts, A. Plenevaux, C. Brihaye and A. Luxen, J. Label. Compd. Radiopharm., 2002, 45, 435-447.

24. S. S. Farn, Y. H. Yeh, W. J. Lin and L. H. Shen, Nucl. Med. Biol., 2009, 36, 225-231.

25. S. J. Archibald, N. Pamme, N. J. Brown and M. D. Tarn, J. Nucl. Med., 2015, 56, suppl. 3, 167.

26. M. D. Tarn, N. J. Brown, N. Pamme and S. J. Archibald, J. Label. Compd. Radiopharm., 2015, 58, S20.

27. M. D. Tarn, D. Maneuski, R. Alexander, N. J. Brown, V. O'Shea, S. L. Pimlott, N. Pamme and S. J. Archibald, Chem. Commun., 2016, 52, 7221-7224.

28. M. P. Taggart, M. D. Tarn, M. M. N. Esfahani, D. M. Schofield, N. J. Brown, S. J. Archibald, T. Deakin, N. Pamme and L. F. Thompson, Lab Chip, 2016, 16, 1605-1616.

29. G. Ribeiro Morais, R. A. Falconer and I. Santos, Eur. J. Org. Chem., 2013, 2013, 1401-1414.

30. F. Svec and Y. Lv, Anal. Chem., 2015, 87, 250-273.

31. G. Guiochon, J. Chromatogr. A, 2007, 1168, 101-168.

32. I. Ali, V. D. Gaitonde and H. Y. Aboul-Enein, J. Chromatogr. Sci., 2009, 47, 432-442.

33. H. Kobayashi, T. Ikegami, H. Kimura, T. Hara, D. Tokuda and N. Tanaka, Anal. Sci., 2006, 22, 491-501.

34. O. Núñez, K. Nakanishi and N. Tanaka, J. Chromatogr. A, 2008, 1191, 231-252.

35. K. K. Unger, R. Skudas and M. M. Schulte, J. Chromatogr. A, 2008, 1184, 393-415.

36. Z. Walsh, B. Paull and M. Macka, Anal. Chim. Acta, 2012, 750, 28-47.

37. C. K. Zacharis, J. Chromatogr. Sci., 2009, 47, 443-451.

38. D. J. Schenk, C. J. Welch and V. Antonucci, J. Label. Compd. Radiopharm., 2016, 59, 391-397.

39. M. Vázquez and B. Paull, Anal. Chim. Acta, 2010, 668, 100113.

40. P. He, B. P. Burke, G. S. Clemente, N. Brown, N. Pamme and S. J. Archibald, React. Chem. Eng., 2016, 1, 361-365.

41. R. Ismail, J. Irribaren, M. R. Javed, A. Machness, R. Michael van Dam and P. Y. Keng, RSC Adv., 2014, 4, 25348-25356.

42. M. Losno, I. Ferrante, R. Brennetot, J. Varlet, C. Blanc, B. Grenut, E. Amblard, S. Descroix and C. Mariet, Micromachines, 2016, 7, 45. 
43. M. Losno, I. Ferrante, R. Brennetot, S. Descroix and C. Mariet, Procedia Chem., 2016, 21, 446-452.

44. A. Bruchet, V. Taniga, S. Descroix, L. Malaquin, F. Goutelard and C. Mariet, Talanta, 2013, 116, 488-494.

45. M. D. Tarn, N. Y. Kızılyer, M. M. N. Esfahani, P. Joshi, N. J. Brown, N. Pamme, D. G. Jenkins and S. J. Archibald, Chem.: Eur. J., 2018, 24, 13749-13753.

46. R. Vasiliadou, M. Nasr Esfahani, N. Brown and K. Welham, Sensors, 2016, 16, 1418.

47. P. He, J. Davies, G. Greenway and S. J. Haswell, Anal. Chim. Acta, 2010, 659, 9-14.

48. P. He, G. Greenway and S. J. Haswell, Microfluid Nanofluid., 2010, 8, 565-573.

49. M. E. Rollie, G. Patonay and I. M. Warner, Ind. Eng. Chem. Res., 1987, 26, 1-6.

50. D. J. Guckenberger, T. E. de Groot, A. M. D. Wan, D. J. Beebe and E. W. K. Young, Lab Chip, 2015, 15, 2364-2378.

51. R. Chand and S. Neethirajan, Biosens. Bioelectron., 2017, 98, 47-53.

52. U. Eletxigerra, J. Martinez-Perdiguero and S. Merino, Sens. Actuator B-Chem., 2015, 221, 1406-1411.

53. J. S. Stefano, R. H. O. Montes, E. M. Richter and R. A. A. Muñoz, J. Braz. Chem. Soc., 2014, 25, 484-491.

54. T. McCreedy, Trends Anal. Chem., 2000, 19, 396-401.

55. "Melting, Boiling, Triple, and Critical Point Temperatures of the Elements", in CRC Handbook of Chemistry and Physics (CD-ROM Version 2010), D. R. Lide (Ed.), CRC Press/Taylor and Francis, Boca Raton, FL, 2010, pp. 133-134.

56. A. W. Bott and B. P. Jackson, Curr. Sep., 1996, 15, 25-30.

57. P. T. Kissinger and W. R. Heineman, J. Chem. Educ., 1983, 60, 702 .

58. R. G. Compton, A. C. Fisher, R. G. Wellington, P. J. Dobson and P. A. Leigh, J. Phys. Chem., 1993, 97, 10410-10415.

59. J. Booth, R. G. Compton, J. A. Cooper, R. A. W. Dryfe, A. C. Fisher, C. L. Davies and M. K. Walters, J. Phys. Chem., 1995, 99, 10942-10947.

60. C. Amatore, N. Da Mota, C. Sella and L. Thouin, Anal. Chem., 2007, 79, 8502-8510.

61. Dionex Technical Note 20: Analysis of carbohydrates by high-performance anion-exchange chromatography with pulsed amperometric detection (HPAE-PAD), Dionex Corporation, 2004.

62. M. Pasta, F. La Mantia and Y. Cui, Electrochim. Acta, 2010, 55, 5561-5568.

63. D.-W. Hwang, S. Lee, M. Seo and T. D. Chung, Anal. Chim. Acta, 2018, 1033, 1-34.

64. Dionex ED4O Electrochemical Detection Operator's Manual, Document No. 034855, Chapter 6.4.3, Revision 3 edn., Dionex Corporation, 1995.

65. D. C. Johnson and W. R. LaCourse, Anal. Chem., 1990, 62, 589A-597A.

66. Dionex Technical Note 21: Optimal settings for pulsed amperometric detection of carbohydrates using the Dionex ED40 electrochemical detector, Dionex Corporation, 1998.

67. A. W. Bott, Curr. Sep., 1998, 17, 25-31.

68. ESA Coulochem III - Application Note: Fluorodeoxyglucose, ESA, Inc., Chelmsford, MA.
69. P. Ullucci, B. Bailey, M. Plante, C. Crafts and I. Acworth, Determination of FDG and CIDG with pulsed amperometric detection on a gold electrode, Thermo Scientific, 2012.

70. M. B. Jensen and D. C. Johnson, Anal. Chem., 1997, 69, 1776-1781.

71. W. R. LaCourse and D. C. Johnson, Anal. Chem., 1993, 65, 50-55.

72. C. M. Zook and W. R. LaCourse, Curr. Sep., 1995, 14, 48-51.

73. , "Guidance for Industry - Q2B Validation of Analytical Procedures: Methodology", available from https://www.fda.gov/regulatory-information/search-fdaguidance-documents/q2b-validation-analyticalprocedures-methodology, U.S. Food and Drug Administration (FDA), Rockville, MD, 1996.

74. C. D. García, G. Engling, P. Herckes, J. L. Collett and C. S. Henry, Environmental Science \& Technology, 2005, 39, 618623.

75. C. D. García and C. S. Henry, Anal. Chem., 2003, 75, 47784783.

76. J. C. Fanguy and C. S. Henry, Analyst, 2002, 127, 10211023.

77. C. D. Garcia and C. S. Henry, Anal. Chim. Acta, 2004, 508, 19.

78. B. Bailey, P. Ullucci, M. Plante, C. Crafts and I. Acworth, Carbohydrate analysis using HPLC with PAD, FLD, Charged Aerosol Detection, and MS detectors, Thermo Scientific.

79. W. R. LaCourse and D. C. Johnson, Carbohydrate Research, 1991, 215, 159-178.

80. Application Note (\#217_038_08) - Clinical \& Diagnostic: Fluorodeoxyglucose, $\left[{ }^{18}\right.$ F]FDG, Radiotracer Analysis, Antec Scientific, available from: https://antecscientific.com/downloads/notes/pharma/217 _038_08\%20\%2OFDG\%2OFluorodeoxyglucose\%20USP\%20EP.pdf, accessed December 2019.

81. A. I. Anzellotti, A. R. McFarland, D. Ferguson and K. F. Olson, Curr. Org. Chem., 2013, 17, 2153-2158.

82. C. D. García and C. S. Henry, Electroanalysis, 2005, 17, 223230.

83. S. Cheung, J. Ly, M. Lazari, S. Sadeghi, P. Y. Keng and R. M. van Dam, J. Pharmaceut. Biomed., 2014, 94, 12-18.

84. N. S. Ha, J. Ly, J. Jones, S. Cheung and R. M. van Dam, Anal. Chim. Acta, 2017, 985, 129-140.

85. C. Zhai, C. Li, W. Qiang, J. Lei, X. Yu and H. Ju, Anal. Chem., 2007, 79, 9427-9432. 